\title{
Lectores de documentos electrónicos
}

\author{
Por Eloísa Monteoliva, Carlos Pérez-Ortiz y Rafael Repiso
}

\begin{abstract}
Resumen: Se repasa la evolución histórica de los dispositivos lectores de documentos electrónicos (EReaders), y se describen las tecnologías del llamado papel electrónico. Se analizan el Gyricon, las pantallas electroforéticas y las que utilizan el principio de electrohumedecimiento. Se presenta la variedad de formatos de archivo compatibles con los distintos dispositivos, así como los problemas que generan. Se describen los principales modelos disponibles en el mercado y sus prestaciones, y se invita a la reflexión sobre su uso y efectividad, la difusión de documentos, así como sobre las expectativas creadas por los e-lectores que, a día de hoy, ya hacen posible la lectura ágil y cómoda de documentos electrónicos.
\end{abstract}

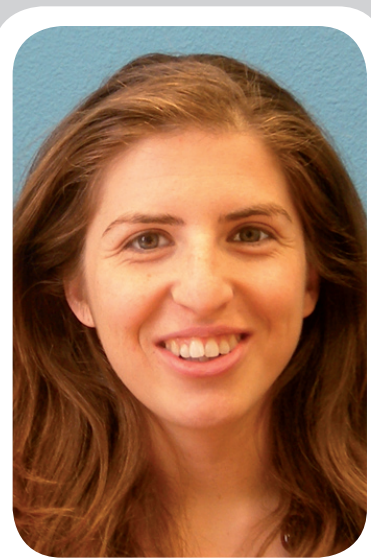

Eloísa Monteoliva-García, es licenciada en traducción e interpretación, especializada en la traducción jurídica, económica y comercial e interpretación de conferencias en inglés, alemán y francés. Investiga la interpretación en los servicios públicos. Es traductora, intérprete y profesora de inglés en ESCO.

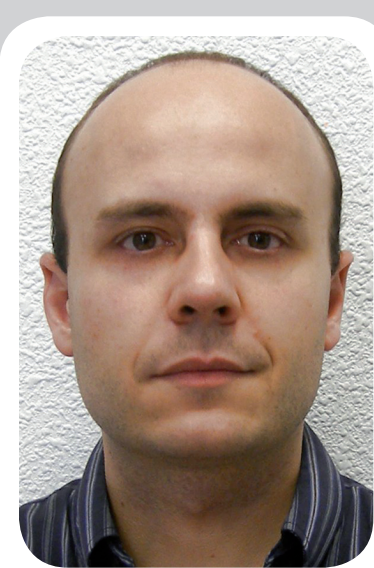

Carlos Pérez-Ortiz, es licenciado en traducción e interpretación. Sus especialidades son la traducción de textos científicos y técnicos, y sus lenguas de trabajo el inglés y el francés. Es director del departamento del Dpto. de Relaciones Externas de ESCO desde 2004, así como traductor y revisor freelance.

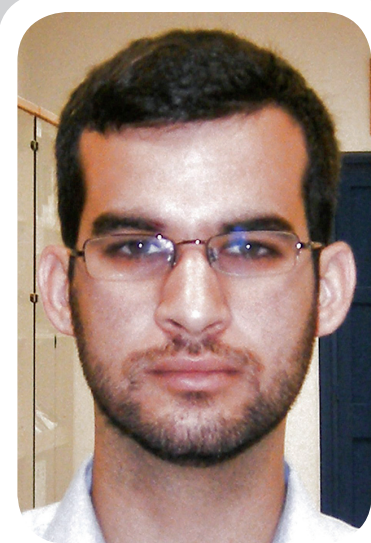

Rafael Repiso-Caballero, es licenciado en documentación por la Universidad de Granada. Becario del Grupo EC3, ha participado en los proyectos In-Recs e In-Reci. Es documentalista de la Escuela Superior de Comunicación (ESCO), Granada.

Palabras clave: Libro electrónico, Biblioteca digital, EReader, E-Book, Documento electrónico, Tinta electrónica, Lectura.

\section{Título inglés: Electronic document readers}

Abstract: This is both a historical overview of reader devices of electronic documents and a description of the current technology characterized by the so called "electronic paper". Gyricon technologies are analysed as well as the electrophoretic screens and devices that use the electrowetting principle. A wide variety of file formats compatible with the different kinds of reader devices are shown, as well as the problems derived from reader incompatibility. The more outstanding models available in the market and their services are also described. A reflection is proposed about the different usages and their effectiveness, document dissemination, and the expectations that the e-readers have created regarding future development. Nowadays these devices make the reading of electronic documents easier and more comfortable.

Keywords: Ebook, Digital library, Electronic document; Electronic ink, Reading, Kindle, Hanlin.

Monteoliva, Eloísa; Pérez-Ortiz, Carlos; Repiso, Rafael. "Lectores de documentos electrónicos”. En: El profesional de la información, 2008, julio-agosto, v. 17, n. 4, pp. 396-402.

DOI: 10.3145/epi.2008.jul.04

\section{Introducción}

Dentro del mundo bibliotecario los dispositivos lectores de documentos electrónicos (e-readers)* no deberían ser un paso más de la evolución de la técnica, ni siquiera herramientas en la que apoyarse puntualmente, sino un componente imprescindible en el desarrollo e implantación de la biblioteca digital. En ésta se fomenta el acceso a una cantidad inmensa de información, la cual se consulta a través de una pantalla, cosa que además previene el gasto innecesario de papel, y por tanto la deforestación de nuestro planeta.
Sin embargo la realidad es que la mayoría de usuarios que necesitan acceder a un documento electrónico no se sienten cómodos leyéndolo en la pantalla del ordenador, y muy probablemente acabarán imprimiendo parte o la totalidad del mismo. Poco a poco, los dispositivos van mejorando y permitiendo una lectura más agradable, son ligeros y cada vez de precio más asequible. Con tales características se hacen idóneos para incorporarse a la biblioteca digital, y no es descabellado pensar en el préstamo de aparatos lectores en bibliotecas o que éstas promuevan su adquisición y uso. 


\section{Antecedentes}

Los orígenes del "lector de libros electrónicos" deben buscarse a finales de la década de 1960, cuando Alan Kay formuló el concepto de Dynabook ${ }^{1}$, un ordenador personal interactivo tan accesible como un libro. Sin embargo, pasarían más de veinte años hasta que Apple Computer desarrollara y comercializara el Apple Newton, dotado de pantalla táctil, que mostraba textos en formato NewtonBook. En aquellos mismos días, Sony lanzó el Data Discman, pronto superado por el Bookman, que después produciría Franklin. Este modelo, pensado sobre todo para la consulta de obras de referencia, no gozó de gran popularidad, debido sobre todo a su pequeña pantalla y lo incómodo de su lectura.

Todos estos modelos quedaron arrinconados ante la facilidad de uso, variedad de prestaciones y comodidad de lectura de las PDA (personal digital assistant, como se recordará), o agendas electrónicas, que contaron con una mayor aceptación por parte de los usuarios. Éstos comenzaron a ver posible la lectura y consulta de textos en aparatos electrónicos que, si bien tenían un tamaño de pantalla reducido, permitían almacenar una gran cantidad de información y proporcionaban una experiencia de lectura algo más agradable gracias a mayores contraste y resolución ${ }^{2}$.

Pero aún quedaba un largo camino por recorrer en la búsqueda de un sustituto o complemento electrónico del libro tradicional. En 1998 Nuvomedia presentó el Rocket eBook, dotado de 4 MB de memoria y una pantalla táctil en blanco y negro de 106 puntos por pulgada $^{3}$. Por su parte, Soft Book Press lanzó al mercado el SoftBook, con una capacidad de memoria notablemente mayor y una pantalla táctil capaz de mostrar 16 tonos de gris. Además, permitía la descarga de contenidos mediante conexión telefónica, servicio que sólo

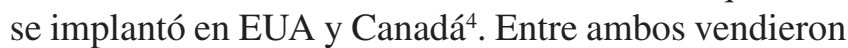
menos de 50.000 unidades. Ninguno de esos modelos se comercializa actualmente, pero sin duda labraron el camino a otros lectores que renunciaban a funciones adicionales para centrarse específicamente en los libros electrónicos.

En el cronograma de la figura 1 se pueden apreciar los hitos que han marcado la historia de los libros electrónicos.

\section{Tecnologías}

Los aparatos lectores almacenan los documentos en memorias internas y tarjetas de memoria, siendo las SD las más usadas, con capacidad para almacenar miles de libros. Suelen tener conexión USB y algunos se conectan inalámbricamente. Pero el gran avance vivido por los lectores de libros electrónicos se debe a la llegada a los mercados de la tecnología que permite una experiencia de lectura agradable, similar a la del papel. Son pantallas pasivas, es decir, no emiten luz propia, que se pueden leer en un rango amplio de condiciones de luz ambiental, sin que dependa del ángulo de visión como las precursoras que usaban pantallas LCD. Tienen un alto contraste, un consumo mínimo (la pantalla no necesita energía para mantener el texto visible, por lo que el gasto es cero hasta que se cambia de página). Las pantallas tienen un grosor de unos $3 \mathrm{~mm}$.

Se han llevado a cabo diversas aproximaciones tecnológicas, de las cuales las más significativas son la tinta electrónica Gyricon, las pantallas electroforéticas y las que aplican el principio de electrohumedecimiento.

\section{"La segunda generación de lectores se caracteriza por el abandono de las pantallas LCD y el uso de E-Ink"}

\section{Gyricon}

Esta tinta electrónica fue desarrollada por Nicholas K. Sheridon en 1974 en el centro de investigación

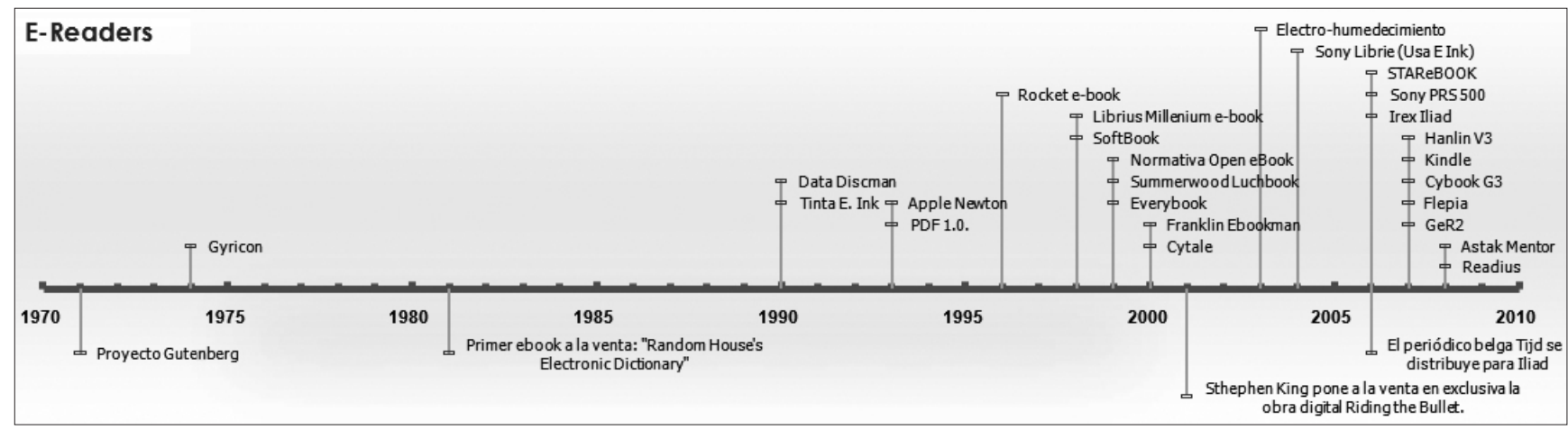

Figura 1. Cronograma de los libros-e (eReaders).

(C) Rafael Repiso 
de Xerox en Palo Alto, California. Consiste en una fina lámina plástica que contiene una serie de diminutas esferas que rotan dentro de una cápsula llena de aceite. Cada hemisferio tiene un color y una carga eléctrica, lo que permite crear diseños mediante la activación selectiva de cada una de las esferas (fig. 2). Xerox clausuró el proyecto Gyricon en 2005 por causas financieras ${ }^{5}$.

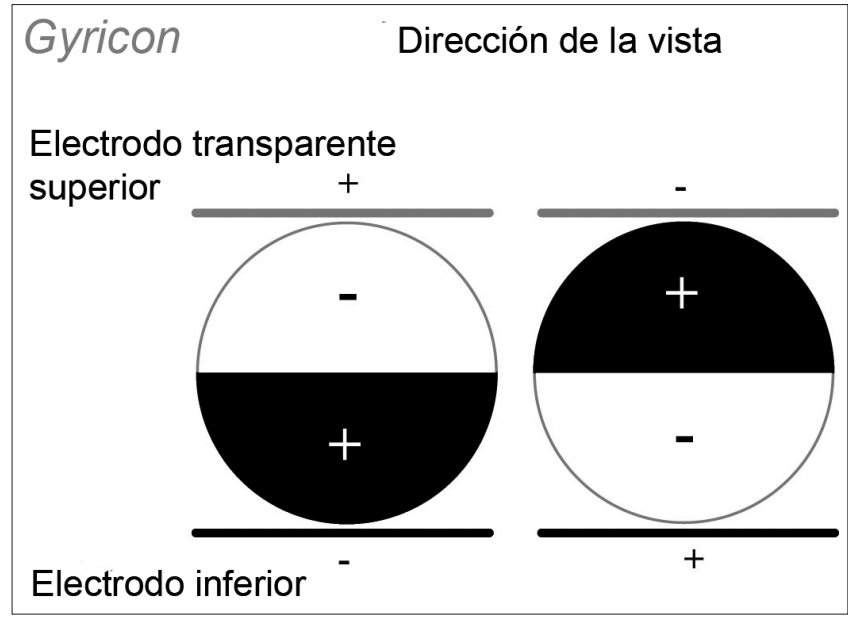

Figura 2

(c) 2003 by Lawrence Erlbaum Ass. Modificada por Rafael Repiso

\section{Pantallas electroforéticas $^{6,7}$}

Emplean multitud de diminutas cápsulas del diámetro aproximado de un cabello humano. Las cápsulas contienen un líquido transparente en el que flotan partículas blancas con carga positiva y negras con carga negativa. La estimulación eléctrica provoca la alineación de las partículas en la parte delantera (visible) o trasera (oculta) de la pantalla, lo que permite crear imágenes en escala de grises con una alta resolución y contraste.

E-ink Corp proporciona estas pantallas a la mayor parte de fabricantes de dispositivos lectores de documentos electrónicos (iLiad, Librié, Sony Reader, Amazon Kindle y HanLin eBook).

\section{"Con E-Ink cada letra se forma con decenas de micro-esferas"}

\section{Electrohumedecimiento}

Logra imágenes de muy alta resolución con tiempos de actualización considerablemente menores. Para ello utiliza la alteración eléctrica del grado de electrofobicidad de un material. Dentro de cada una de las células se encuentra una cierta cantidad de aceite coloreado y otra de agua (fig. 4). Ésta, repelida naturalmente por el material hidrófobo, tiende a moverse y aumentar su superficie de contacto con este material cuando se aplica a éste último un potencial eléctrico, cambiando así el patrón visible en un tiempo reducido y con una alta resolución. Liquavista, empresa surgida de la holandesa Philips, y la alemana $A D T$, trabajan en la aplicación de esta tecnología a diversos dispositivos, entre los que se cuentan monitores y teléfonos móviles ${ }^{8}$.

\section{Formatos}

Los dispositivos que están en el mercado aceptan distintos formatos de texto e imagen. Algunos de

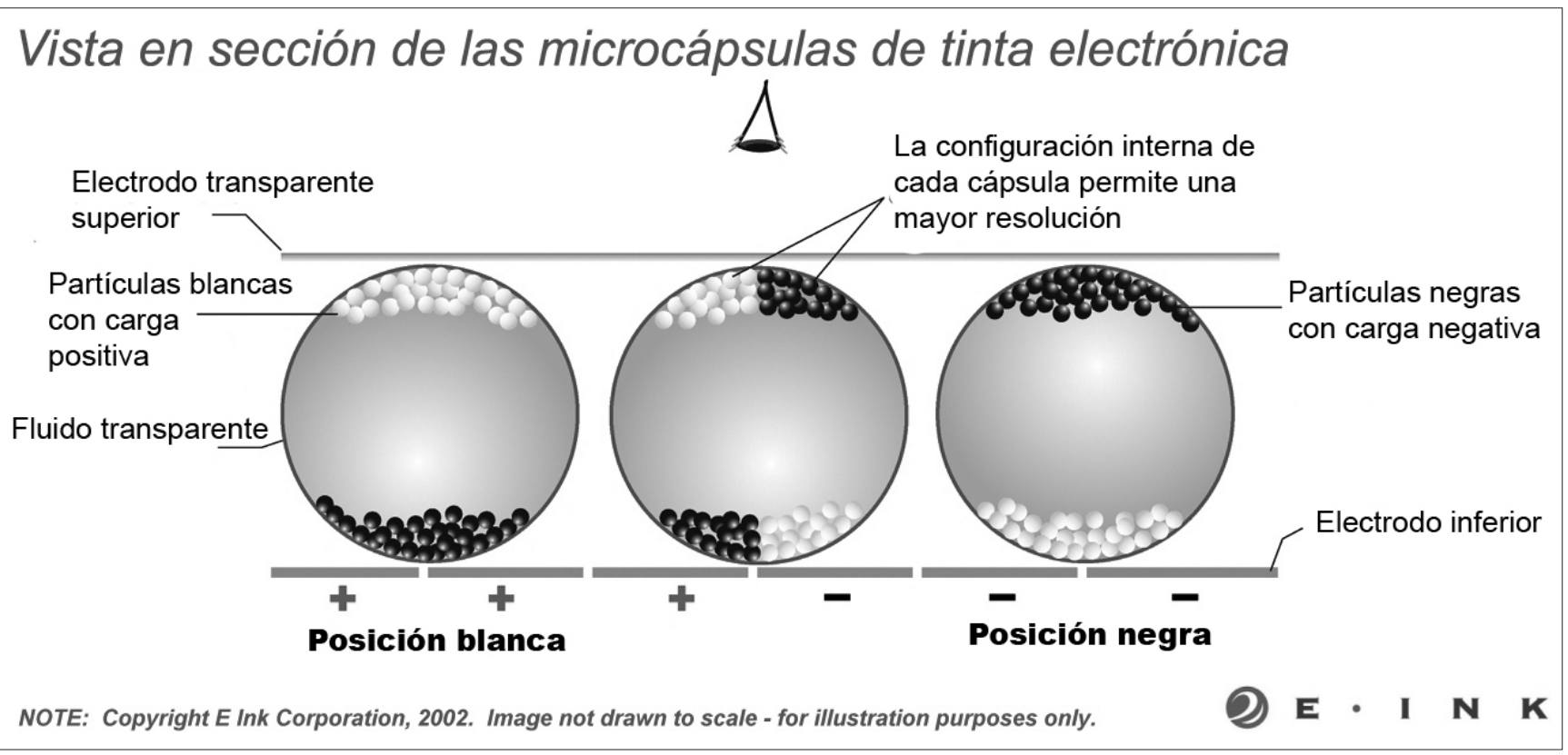




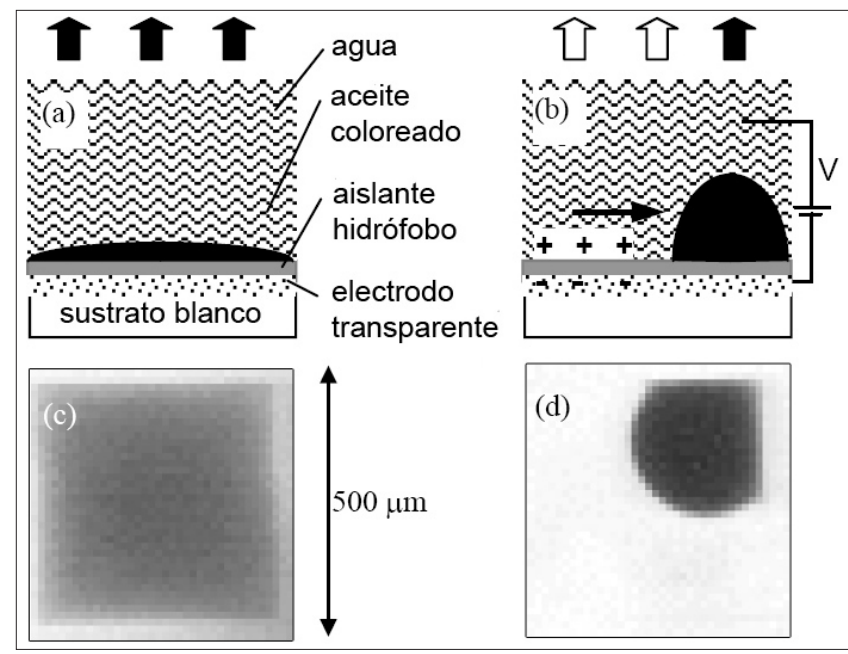

Figura 4. Principio de funcionamiento de la pantalla de electrohumedecimiento. Esquema en sección: (a) sin aplicar tensión presenta una película homogénea de aceite; (b) aplicando corriente continua se causa una contracción del aceite. Las fotografías en vista superior (c) y (d) muestran como el aceite se retrae ante un electrodo.

(C) Philips Res. Lab. Modificada por Rafael Repiso

ellos son exclusivos para una marca determinada (.azw, .BBeB, .rb, .stk); otros son compatibles entre distintos lectores (.djvu, .doc, .fb2, .gif, .html, .jpg, .mobi, .pdf, .png, .rar, .rtf, .txt, .zip). También hay que distinguir si tienen o no restricciones de gestión de derechos digitales o DRM (digital rights management) impuestas por la marca.

Los formatos afectan tanto a la oferta de documentos electrónicos disponibles como a la posibilidad de transferirlos de unos dispositivos a otros. Existen numerosos formatos en el mercado que pueden inducir a los usuarios a confusión, pues pueden descargar o comprar uno que su dispositivo no lea. La diversidad de formatos incompatibles entre sí perjudica al mercado y a los consumidores. Lo ideal sería tender hacia un número más reducido de ellos, que asegure su compatibilidad con todos los dispositivos, de tal modo que se alcance lo que se conoce como "interoperabilidad", es decir, el intercambio de archivos de formatos compatibles entre unos dispositivos y otros.

Una dificultad con que se encuentran los eReaders es la existencia de libros tanto nacidos digitales como digitalizados a base de escanear versiones en papel ${ }^{9}$. La tendencia actual es digitalizar los libros manteniendo el formato original del libro y permitiendo opciones OCR (reconocimiento de caracteres). Esto obliga a que los aparatos tengan que poder ajustar las páginas a sus propias características físicas. Para subsanar este problema existen programas de conversión.

La cuestión de la interoperabilidad guarda relación con el DRM. Los derechos pueden determinar que un archivo sea legible en un dispositivo y no en otro, independientemente de que el formato sea compatible.
El DRM impone una serie de limitaciones que reducen la interoperabilidad de los archivos y, por otro lado, no se ha demostrado su eficacia para luchar contra la piratería.

Esto es defendido por el estadounidense David Rothman, autor del blog sobre e-books Teleread.org, quien dice además que el $D R M$ perjudica al consumidor. Ha lanzado un llamamiento a los editores para que opten por el denominado "DRM social", una idea propuesta por el director general de Adobe ePublishing, Bill McCoy, según la cual la única restricción técnica sería la presencia de un sello en los archivos que diría "Este e-book es propiedad de...", lo cual ampliaría el mercado de documentos electrónicos y evitaría que un solo actor tenga el monopolio del mercado ${ }^{10}$.

A continuación presentamos un resumen de las características de los principales formatos propietarios:

- AZW: se trata del formato Kindle y consiste básicamente en una ligera modificación de Mobipocket a la que se añade el $D R M$. Es el único que se puede descargar de Amazon. Los archivos Mobi sin DRM se pueden leer en Kindle.

- BBeB: Broadband Ebook, propietario de Sony, encriptado con DRM para su Sony Reader.

- Epub: abierto basado en XML, legible para distintas soluciones de software para e-books, tanto para Windows como para Linux, PocketPC y Palm OS. Lo utilizan HaaliReader, FBReader y PalmFiction. Fue lanzado por el International Digital Publishing Forum (antes Open eBook Forum), el grupo comercial de publicaciones digitales. Sus principales virtudes son la posibilidad de convertirlo a un gran número de formatos para distintos dispositivos de lectura, y su capacidad de "reajuste", es decir, de adaptar su tamaño al de la pantalla del lector. Entre otros, lo han adoptado Penguin, HarperCollins y Random House. Probablemente Sony Reader será compatible con epub cuando salga al mercado británico a finales de este año 2008.

- MOBI: Fundado en el año 2000, Mobipocket se ha convertido en el principal formato para dispositivos móviles, como PDAs y Blackberry. La empresa fue absorbida por Amazon en 2005. El software Windows PC Mobipocket puede convertir archivos .chm, .doc, .html, .ocf, .pdf, .rtf y .txt a este formato.

- RB: formato para Rocket eBook y Gemstar RCA REB 1100.

- STK: formato de STAReBOOK

\section{Actualidad}

Como se puede observar en la tabla 1 , después de un periodo de desarrollo de más de tres décadas actualmente nos encontramos ante un mercado marcado por 
el uso de la tecnología E-Ink. La generación actual de dispositivos lectores de documentos electrónicos está caracterizada por la diversificación de la oferta, por un aumento de la funcionalidad de los mismos y por un abaratamiento del coste de los e-books, de tal modo que el producto es más accesible y asequible para los usuarios.

\section{"La tinta electrónica E-Ink no emite luz, y ésta quizá sea la clave de su éxito"}

Tanto los usuarios que busquen un aparato que les permita simplemente leer documentos electrónicos como aquellos que deseen un dispositivo interactivo (que permita insertar notas, subrayar, etc.) encuentran distintas opciones de compra. El mayor o menor coste de los mismos dependerá de sus prestaciones (acceso a internet, subrayado, búsquedas, etc.) y no estará relacionado con una mayor o menor calidad de visualización.

En lo que respecta a los documentos electrónicos, actualmente la tipología se está ampliando, puesto que encontramos documentos dirigidos a distintos ti-

Tabla 1. Comparación de características de aparatos lectores de documentos-e (eReaders). (C) 2008, Rafael Repiso.

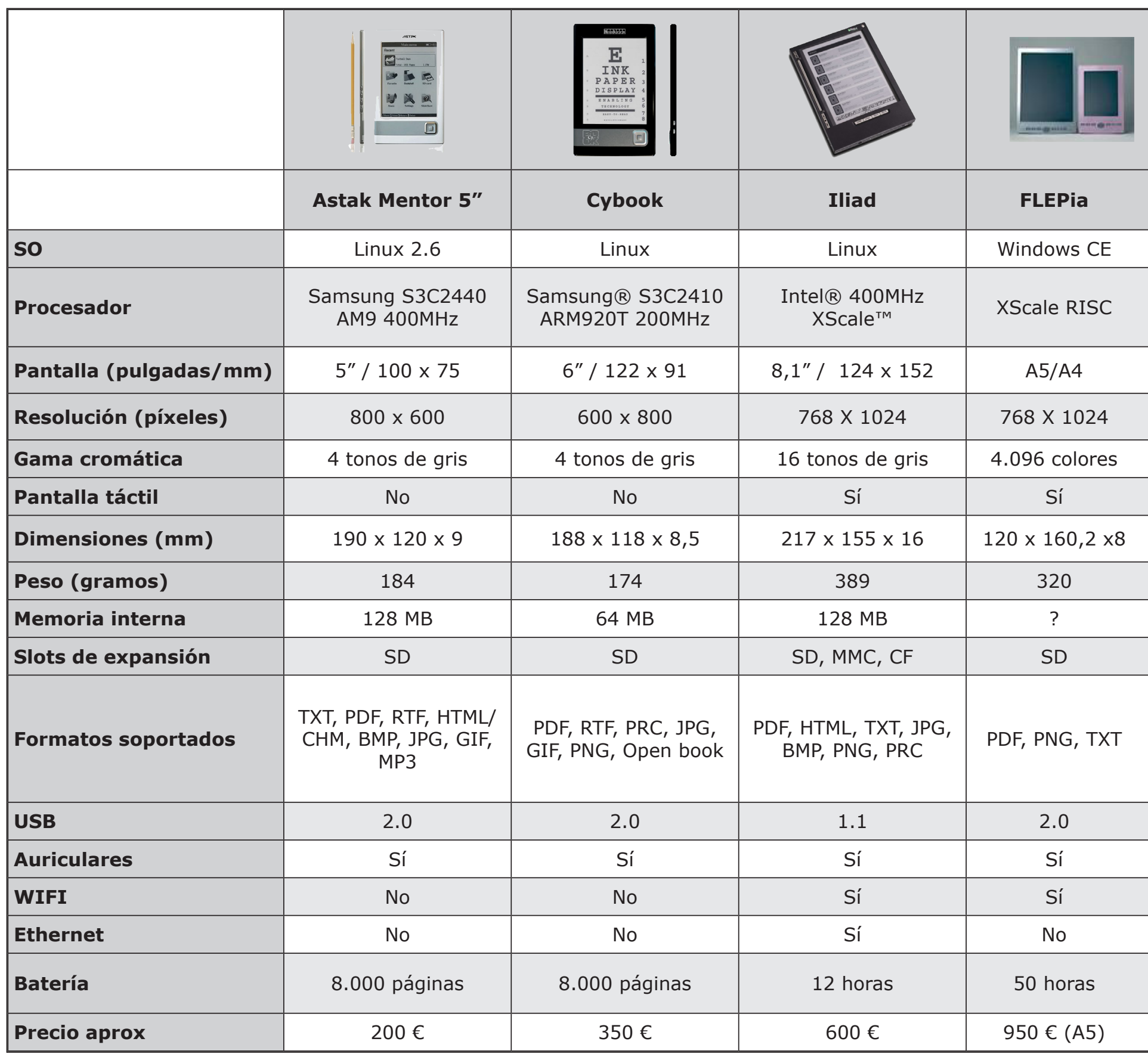

* El Hanlin v.3 en su última actualización permite abrir archivos mobi, y se espera que en la próxima abra ppt. 
pos de lectores, desde puramente científicos y académicos, manuales, guías, hasta una amplia variedad de literatura no científica. Además estamos presenciando un proceso de implantación de documentos electrónicos en sistemas de venta y en sistemas de información digital: aulas, diarios, bibliotecas y depósitos gratuitos.

\section{Conclusiones}

No cabe duda de que en el futuro estos dispositivos no permitirán simplemente la lectura de documentos electrónicos, sino también la gestión de los mismos.
Actualmente los usuarios pueden conectarse a internet, pero las prestaciones que ofrezcan los dispositivos de lectura deben tender hacia la interacción con el usuario, la cual vendría determinada por:

- Navegación rápida y eficaz por los distintos documentos. La navegación óptima será aquella que haga posible realizar una consulta eficiente mediante el uso de libros de trabajo (CDU, Biblia, etc.).

- Interactividad del lector: que éste pueda subrayar, escribir notas marginales, compartir, etc.

- Inclusión de un motor de búsqueda que permita

\begin{tabular}{|c|c|c|c|c|}
\hline Hanlin V3 & Kindle & Readius & Sony Reader 505 & $\begin{array}{c}\text { STAR } \\
\text { eBOOK }\end{array}$ \\
\hline Linux & Marvell PXA255 & Windows & Linux & Linux \\
\hline $\begin{array}{c}\text { Samsung Arm9 } \\
200 \mathrm{Mhz}\end{array}$ & Marvell PXA255 & ARM11 400MHz & No facilitado & $\begin{array}{c}\text { Samsung Arm } \\
250 \mathrm{MHz}\end{array}$ \\
\hline $6 " / 90 \times 120$ & $6 " / 122 \times 91$ & $5^{\prime \prime} / 100 \times 75$ & $6 " / 122 \times 91$ & $6 " / 122 \times 91$ \\
\hline $600 \times 800$ & $600 \times 800$ & $320 \times 240$ & $600 \times 800$ & $600 \times 800$ \\
\hline 4 tonos de gris & 4 tonos de gris & 16 tonos de gris & 8 tonos de gris & 4 tonos de gris \\
\hline No & Teclado qwerty & No & No & No \\
\hline $184 \times 120,5 \times 9,9$ & $184 \times 120 \times 10$ & $115 \times 57 \times 21$ & $175 \times 124 \times 12$ & $188 \times 118 \times 8$ \\
\hline 210 & 290 & 115 & 255 & 176 \\
\hline $32 \mathrm{MB}$ & $256 \mathrm{MB}$ & 256 MB & $128 \mathrm{MB}$ & $64 \mathrm{MB}$ \\
\hline SD & SD & Micro SD & SD, Memory stick & SD \\
\hline $\begin{array}{l}\text { PDF, TXT, RTF, DOC, } \\
\text { CHM, FB2, HTML, } \\
\text { DJVU, ZIP, RAR, } \\
\text { TIFF, JPEG, GIF, BMP, } \\
\text { PNG, MP3, MOBI* }\end{array}$ & $\begin{array}{l}\text { AZW, TXT, MOBI, } \\
\text { PRC, MP3, AA }\end{array}$ & PDF, HTML , TXT & $\begin{array}{c}\text { PDF, TXT, RTF, } \\
\text { WORD, JPG, GIF, } \\
\text { PNG, BMP, BBEB } \\
\text { Book }\end{array}$ & STK; MP3, JPG \\
\hline 1.1 & 2.0 & 2.0 & 1.1 & 2.0 \\
\hline Sí & Sí & Sí & Sí & Sí \\
\hline No & Sí & $3 \mathrm{G}$ & No & No \\
\hline No & Whispernet & HSDPA/ $3 G$ & No & No \\
\hline 8.000 páginas & $\begin{array}{l}\text { De } 1 \text { a } 7 \text { días, según } \\
\text { funciones }\end{array}$ & 30 horas & 7.500 páginas & 8.000 páginas \\
\hline $325 €$ & $300 €$ & $600 €$ & No & No \\
\hline
\end{tabular}


al usuario organizar y buscar documentos en su biblioteca digital, que podrá llegar a albergar miles de documentos.

- Reproducción de archivos multimedia.

\section{"El futuro de los dispositivos de lectura pasa por la interactividad con el usuario"}

Las prometedoras tecnologías aplicadas a la fabricación de dispositivos lectores de documentos electrónicos deben superar algunos retos antes de implantarse de forma sólida en un sector amplio del mercado. De sus características deseables cabe destacar que sean delgados y flexibles, que tengan un consumo mínimo entre giros de página, que se lean cómodamente en condiciones diversas de luz ambiental, que muestren una alta resolución de gráficos, que puedan reproducir color y que tengan un fondo lo bastante blanco para generar buen contraste. En cuanto a la duración de la batería, la recarga por energía solar o cinética ofrecen interesantes posibilidades.

\section{Nota}

*Preferimos llamar e-readers a los dispositivos lectores, más que $e$-books, dado que pueden leer muchos tipos de documentos.

\section{Referencias bibliográficas}

1. Kay, Alan; Goldberg, Adele. "Personal dynamic media". En: Computer, 1977, v. 10, n. 3, pp. 31-41.

2. PDA eBooks

http://www.writers-publish.com/pda-ebooks.html

3. Senso, Jose A. Libros electrónicos al alcance de la mano. En: El profesional de la información, 1998, nov., v. 7, n. 11, pp. 32-33.

http://www.elprofesionaldelainformacion.com

4. Coyle, Karen. "E-Reading." En: The journal of academic librarianship, 2008, marzo, v. 34 , n. 2, pp. $160-162$.

5. Genuth, Iddo. "The future of electronic paper". En: The future of things.

http://www.tfot.info/articles/1000/the-future-of-electronic-paper.html

6. Jacobson, J.; Comiskey, B.; Turner, C.; Albert, J.; Tsao, P. "The last book". En: IBM systems journal, 1997, v. 36, n. 3, pp. 457-463.

7. Desmarais, Norman. "Innovations affecting us - E-Ink and digital paper”. En: Against the grain. 2002, v. 14, n. 6, pp. 87-90.

8. Hayes, Robert A.; Feenstra, Bokke J.; Camps, Ivo G. J.; Hage, Leendert; Roques-Carmes, M. T.; Schlangen, L. J. M. et al. "A high brightness colour 160 ppi reflective display technology based on electrowetting". En: SID Symposium digest of technical papers, 2004, pp. 1412-1415.

9. Chvatik, Daniel. "SoftBook reader". En: ATPM, 2000, mayo, v. 6, n. 5, pp. 123-130

10. Tivnan, Tom. eBabel on and on

http://www.thebookseller.com/in-depth/feature/59816-ebabel-on-and-on.$h t m l$

Eloísa Monteoliva,emonteoliva@escogranada.com Carlos Pérez-Ortiz, rexternas@escogranada.com Rafael Repiso,rrepiso@escogranada.com Escuela Superior de Comunicación (Centro asociado a la Universidad de Gales), San Antón, 73. 18005 Granada.

\section{Te damos los ingredientes...}

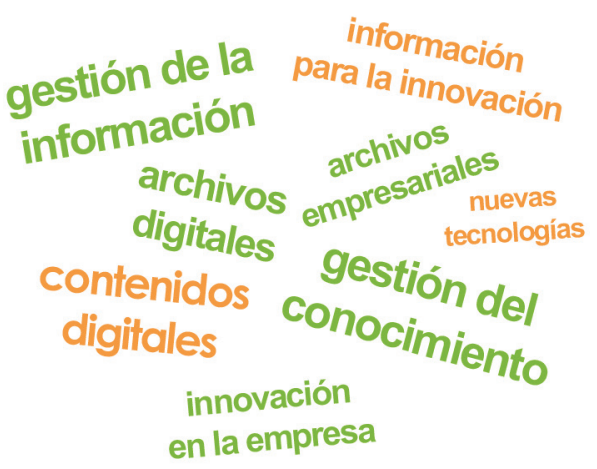

para que elabores el plato

El profesional de la información

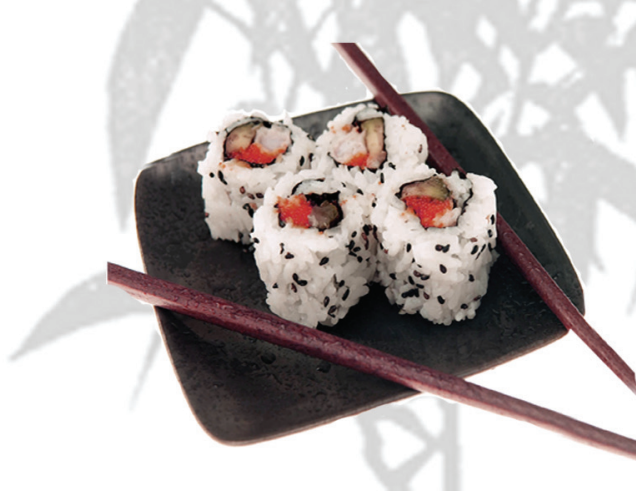

